\title{
Robust adaptive control of coaxial octorotor UAV using type-1 and interval type-2 fuzzy logic systems
}

\author{
Hemza Mekki ${ }^{1 *}$, Ali Djerioui ${ }^{1}$, Samir Zeghlache ${ }^{2}$, Abderrahmen Bouguerra ${ }^{1}$ \\ ${ }^{1}$ LGE, Laboratoire de Génie Electrique, Department of Electrical Engineering, Faculty of Technology, Mohamed Boudiaf \\ University of Msila, BP 166 Ichbilia, Msila, Algeria \\ ${ }^{2}$ LASS, Laboratoire d'Analyse des Signaux et Systèmes, Department of Electrical Engineering, Faculty of Technology, \\ Mohamed Boudiaf University of Msila, BP 166 Ichbilia, Msila, Algeria
}

Corresponding Author Email: hemza.mekki@g.enp.edu.dz

https://doi.org/10.18280/ama_c.730405

Received: 19 March 2018

Accepted: 25 June 2018

\section{Keywords:}

fuzzy system, adaptive control, robust control, coaxial octorotor

\begin{abstract}
In this paper, a robust controller for a Six Degrees of Freedom (6 DOF) coaxial octorotor unmanned aerial vehicle (UAV) control is proposed in presence of the disturbances and uncertainties. Adaptive control theory based on type-1 and interval type-2 Fuzzy inference systems is used to design a controller for each subsystem of the octorotor helicopter. The proposed control scheme allows avoiding the difficult modeling, guaranteeing the stability and the robustness of the system. Exponential stability of the closed loop is guaranteed by using Lyapunov theory. The performance and the effectiveness of the proposed controller, simulation results are confirmed by simulation study.
\end{abstract}

\section{INTRODUCTION}

Helicopters are dynamically unstable and therefore suitable control methods are needed to stabilize them. In order to be able to optimize the operation of the control loop in terms of stability, precision and reaction time, it is essential to know the dynamic behavior of the process which can be established by a representative mathematical model.

However, in practical situations, there are many difficult problems in controlling multirotors helicopters because of the inevitable uncertainties. Thus many efforts have been made to control quadrotor helicopter and some strategies have been developed to solve the path following problems for this type of system. In [1], a PID controller which can perform steady flight of quadrotor helicopter from one point to anther has been designed.

Sliding mode control has been extensively tested to control quadrotors systems. The advantage of this approach is its insensitivity to the model errors and parametric uncertainties, as well as the ability to globally stabilize the system in the presence of other disturbances [2]. The sliding mode controller designed in [3] improves robustness of the external disturbances and model uncertainty of quadrotor system. In [4] backstepping approach was used to control quadrotor system where some parametric and non-parametric uncertainties are considered. In [5] a fuzzy controller based on on-line optimization of a zero order Takagi-Sugeno fuzzy inference system, which is tuned by a back propagation-like algorithm, is applied to a quadrotor. Where some uncertainties are considered. The work presented in [6] proposes an adaptive neural network control to stabilize a quadrotor helicopter against modeling error and wind disturbance.

The robust control methods proposed in the literature are all based on the inherent structure of quadrotor that leads to the deficiency in driving capability. A novel coaxial eight-rotor
UAV model proposed in [7] has been used in this paper. It is designed with eight rotors that are arranged as four counter rotating offset pairs mounted at the ends of four carbon fiber arms in a cruciform configuration. In addition, the coaxial octorotor UAV can be designed to accommodate when some of motors fail and still permit to the helicopter to continue flying.

The main contribution provided in this paper consists of the developpement adaptive and robust controller for a class of systems encountered mainly in aerial vehicles. The mathematical model of the aerial vehicles can be regarded as the association of several interconnected subsystems. First, the type-2 fuzzy systems are utilized to approximate the local nonlinearities of each subsystem, and then the fuzzy parameters are on-line adjusted by adaptive laws with stability and convergence analysis of the Lyapunov theory in order to achieve the expected tracking performance. The robustcontrol terms based on type-1 fuzzy systems are added in order to attenuate the influence of external disturbances, interconnection effects between subsystems and the type-2 fuzzy approximation error. It should be noted that the advantage of decentralized control design is to reduce complexity and this allows the control implementation to be more feasible and in addition the computing time is reduced. In order to show the effectiveness of the proposed approach, simulation studies of the control scheme are carried out using the coaxial octorotor helicopter dynamical model. In addition the proposed control scheme allows to avoid the nonlinear modeling problems, guarantee the stability of the helicopter in closed loop, to provide the robustness and to obtain the desired trajectory tracking with best performances.

The rest of paper is organized as follow. First, the problem formulation is presented in section 2. A brief description of interval type-2 fuzzy logic system (IT2FLS) is then introduced in section 3. Adaptive type-1 and interval type-2 fuzzy 
controller design for MIMO systems is given in section 4. In section 5 and 6 , the proposed scheme performances are evaluated by simulation in the case of the coaxial octorotor helicopter. Finally conclusions on the present paper are driven.

\section{PROBLEM FORMULATION}

Consider a class of nonlinear MIMO dynamic systems that is encountered mainly in the multirotor UAV field described the following differential equations:

$$
\sum_{i}\left\{\begin{array}{l}
x_{i}^{\left(r_{i}\right)}=F_{i}\left(\bar{x}_{i}\right)+\bar{g}_{i}\left(\bar{x}_{i}\right) u_{i}+\Delta_{i}(t) \\
y_{i}=x_{i} \quad i=1,2, \ldots, m
\end{array}\right.
$$

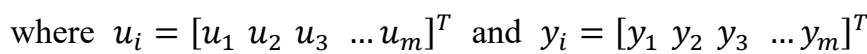
are the control input and output of the system, the functions $F_{i}\left(\bar{x}_{i}\right)$ are continuous nonlinear functions, which are supposed unknown, $\bar{g}_{i}(\bar{x})$ are continuous nonlinear functions, which are supposed unknown, $\bar{x}_{i}=\left[\begin{array}{llllll}x_{i} & \dot{x}_{i} & \ddot{x}_{i} & \ldots & x_{i}^{\left(r_{i}-1\right)}\end{array}\right]^{T}$ are the states variables, $r_{i}$ are positive integers $i=1,2, \ldots, m$ which means the order time derivative of the variable $x_{i}$, and $\Delta_{i}(t)$ are the external disturbances and uncertainties. The control objective to force the system output $y_{i}$ to follow the given reference signal $y_{d i}$ under external disturbances and uncertainties. In order to attain this objective, some assumptions have been introduced.

Assumption1. $\bar{g}_{i}(\bar{x})$ are continuous nonlinear functions, which are supposed unknown.

Assumption2. The external disturbances and uncertainties $\Delta_{i}(t)$ are bounded, i.e.,

$$
\sup _{t \geq 0}\left|\Delta_{i}(t)\right| \leq \delta_{i}
$$

where $\delta_{i}$ are known positive constant.

Let us now define the reference signal vector $y_{d i}$ and the tracking error vector $e_{i}$ as follows:

$e_{i}=y_{i}-y_{d i}=\left[e_{i} \dot{e}_{i} \ddot{e}_{i} \ldots e_{i}^{\left(r_{i}-1\right)}\right]^{T}$

The sliding surface is defined as:

$$
s_{i}=c^{T} e_{i}=e_{i}^{\left(r_{i}-1\right)}+c_{r_{i}-1} e_{i}^{\left(r_{i}-2\right)}+\ldots+c_{1} e_{i}
$$

where $c^{T}=\left[\begin{array}{lllll}c_{1} & \ldots & c_{r_{i}-2} & c_{r_{i}-1} & 1\end{array}\right]^{T} \in \mathfrak{R}^{n}$ are the coefficients of the Hurwitz polynomial. If the initial condition $e_{i}(0)=0$ then the tracking problem can be considered as the state error vector remaining on the sliding surface $s_{i}=0$ for all $t \geq 0$. A sufficient condition that the system controlled is stable is given in [2] as:

$$
s_{i} \dot{s}_{i} \leq-\eta_{i}\left|s_{i}\right|, \quad \eta_{i}>0
$$

where $\eta_{i}$ is a constant design parameter. Then sliding condition of (5) can be rewritten as follow:

$$
\dot{s}_{i} \leq-\eta_{i} \operatorname{sgn}\left(s_{i}\right)
$$

where:

$$
\operatorname{sgn}\left(s_{i}\right)=\left\{\begin{array}{l}
1 \text { for } s_{i}>0 \\
0 \text { for } s_{i}=0 \\
-1 \text { for } s_{i}<0
\end{array}\right.
$$

In the case of our work in order to eliminate the chattering effect which can damage the actuators, a continuous type-1 fuzzy logic control (T1FLC) is used to approximate the hitting control $\left(u_{d c i}=\operatorname{sgn}\left(s_{i}\right)\right)$.

where $u_{d c i}$ is the outputs of the T1FLC, which is obtained by the sliding surfaces variables $s_{i}$.

The fuzzy membership functions of the input variables $s_{i}$ and output discontinuous control $u_{d c i}$ sets are presented by Fig.1.

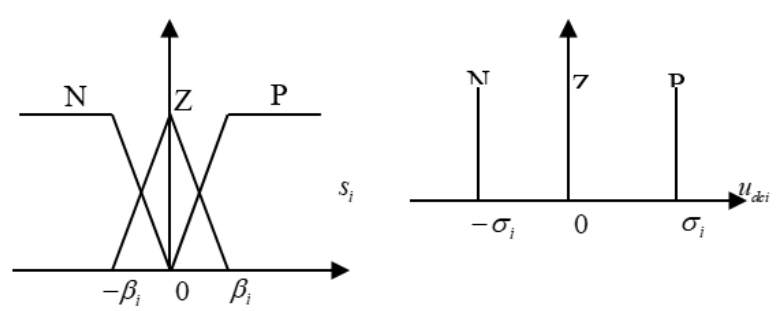

Figure 1. Membership functions of input variables $s_{i}$ and output $u_{f s i}$ for $i=\psi, \varphi$

All the membership functions of the fuzzy input variable are chosen to be triangular. The used labels of the fuzzy variable surfaces $s_{i}$ are: $\{$ negative $(\mathrm{N})$, zero $(\mathrm{Z})$ and positive $(\mathrm{P})$ \}.

The fuzzy labels of the hitting control variables $u_{d c i}$ are a singletons membership functions decomposed into three levels represented by a set of linguistic variables: \{negative $(\mathrm{N})$, zero $(\mathrm{Z})$ and positive $(\mathrm{P})\}$. Table. 2 presents the rules base which contains 3 rules:

Table 1. Rules base [8]

\begin{tabular}{|c|l|l|l|}
\hline & Rule1 & Rule2 & Rule3 \\
\hline$s_{i}$ & $\mathrm{P}$ & $\mathrm{Z}$ & $\mathrm{N}$ \\
\hline$u_{f s i}$ & $\mathrm{P}$ & $\mathrm{Z}$ & $\mathrm{N}$ \\
\hline
\end{tabular}

The hitting control laws are computed by fuzzy logic inference mechanism as [8]:

$u_{d c i}=\operatorname{T1FLC}\left(s_{i}\right)=\frac{\sum_{j=1}^{3} \alpha_{i j} \omega_{i j}}{\sum_{j=1}^{3} \alpha_{i j}}$
$=\alpha_{i 1} \sigma_{i 1}+\alpha_{i 2} \sigma_{i 2}+\alpha_{i 3} \sigma_{i 3}$

where $0 \leq \alpha_{i 1} \leq 1,0 \leq \alpha_{i 2} \leq 1$ and $0 \leq \alpha_{i 3} \leq 1$ are the membership degree of rules 1,2 and 3 presented in Table.1, $\sigma_{i 1}=\sigma_{i}, \sigma_{i 2}=0$ and $\sigma_{i 3}=-\sigma_{i}$ are the center of output membership functions $u_{d c i}$. The relation $\alpha_{i 1}+\alpha_{i 2}+\alpha_{i 3}=1$ is valid according to the triangular membership functions. Moreover, the fuzzy hitting control can be further analyzed as 
the following four conditions, and only one of four conditions will occur for any value of $s_{i}$, according to Fig.1 [8].

\section{Condition 1}

Only rule 1 is activated $\left(s_{i}>\beta_{i}, \alpha_{i 1}=1, \alpha_{i 2}=\alpha_{i 3}=0\right)$

$u_{d c i}=T 1 F L C\left(s_{i}\right)=\sigma_{i}$

\section{Condition 2}

Rule 1 and 2 are activated simultaneously $\left(0<s_{i} \leq \beta_{i}\right.$, $0<\alpha_{i 1}, \alpha_{i 2} \leq 1, \alpha_{i 3}=0$ )

$u_{d c i}=\operatorname{T1FLC}\left(s_{i}\right)=\alpha_{i 1} \sigma_{i 1}=\alpha_{i 1} \sigma_{i}$

\section{Condition 3}

Rule 2 and 3 are activated simultaneously $\left(-\beta<s_{i} \leq 0_{i}\right.$, $0<\alpha_{i 2}, \alpha_{i 3} \leq 1, \alpha_{i 1}=0$ )

$u_{d c i}=\operatorname{T1FLC}\left(s_{i}\right)=\alpha_{i 3} \sigma_{i 3}=-\alpha_{i 3} \sigma_{i}$

\section{Condition 4} 0)

Only rule 3 is activated $\left(s_{i} \leq-\beta_{i}, \alpha_{i 1}=1, \alpha_{i 2}=\alpha_{i 3}=\right.$

$u_{d c i}=T 1 F L C\left(s_{i}\right)=\sigma_{i}$

According to four possible conditions shown in (9)-(12) [8], we obtain:

$u_{d c i}=T 1 F L C\left(s_{i}\right)=\sigma_{i}\left(\alpha_{i 1}-\alpha_{i 3}\right)$

And it can see that

$s_{i} u_{d c i}=s_{i} T 1 F L C\left(s_{i}\right)=\sigma_{i}\left|\alpha_{i 1}-\alpha_{i 3}\right|\left|s_{i}\right|$

The equation (5) can be rewritten by:

$s_{i} \dot{s}_{i} \leq-\eta_{i} \sigma_{i}\left|\alpha_{i 1}-\alpha_{i 3}\right|\left|s_{i}\right|, \quad \eta_{i}>0$

Or

$\dot{s}_{i} \leq-\eta_{i} T 1 F L C\left(s_{i}\right)$

The time derivative of the sliding surface in (4) is computed by:

$\dot{s}_{i}=\sum_{k=1}^{r_{i}-1} c_{k} e_{i}^{k}+x^{r_{i}}-y_{d i}^{r_{i}}$

$=\sum_{k=1}^{r_{i}-1} c_{k} e_{i}^{k}+F_{i}\left(\bar{x}_{i}\right)+\bar{g}_{i}\left(\bar{x}_{i}\right) u_{i}+\Delta_{i}(t)-y_{d i}^{r_{i}}$

Substituting (17) into (16), sliding condition can be reexpressed as:

$$
\begin{aligned}
& \left(\sum_{k=1}^{r_{i}-1} c_{k} e_{i}^{k}+F_{i}\left(\bar{x}_{i}\right)+\bar{g}_{i}\left(\bar{x}_{i}\right) u_{i}+\Delta_{i}(t)-y_{d i}^{r_{i}}\right) \\
& \leq-\eta_{i} \operatorname{T} 1 F L C\left(s_{i}\right)
\end{aligned}
$$

If $F_{i}\left(\bar{x}_{i}\right)$ and $\bar{g}_{i}\left(\bar{x}_{i}\right)$ are known and free of external disturbance and uncertainties, i.e., $\Delta_{i}(t)=0$, the ideal controls law can be obtained as:

$$
u_{i}^{*}=\frac{1}{\bar{g}_{i}(\bar{x})}\left(-\sum_{k=1}^{r_{i}-1} c_{k} e_{i}^{k}-F_{i}\left(\bar{x}_{i}\right)+y_{d i}^{r_{i}}-\eta_{i \Delta} \operatorname{T1FLC}\left(s_{i}\right)\right)
$$

where $\eta_{i_{\Delta}} \geq \eta_{i} \geq 0$.

Sadly, $F_{i}\left(\bar{x}_{i}\right), \bar{g}_{i}\left(\bar{x}_{i}\right)$ are unknown and $\Delta_{i}(t)$ terms which include external disturbance and uncertainties are not null, in our problem, so it is impossible to obtain the control law (19). In order to solve such problems, an adaptive control strategy by the use of type-2 fuzzy systems is proposed. This approach rests on the online estimation of the local nonlinearities $F_{i}\left(\bar{x}_{i}\right)$ and $\bar{g}_{i}\left(\bar{x}_{i}\right)$ using the type- 2 fuzzy systems where the conclusions parameters are adjusted on-line according to some adaptive laws. In this paper, additional robust-control term based on fuzzy type-1 inference system is employed to attenuate both the effects of fuzzy type- 2 approximation error, external disturbance and uncertainties effect

\section{INTERVAL TYPE-2 FUZZY LOGIC SYSTEM}

In this section, the interval type-2 fuzzy set and the inference of type-2 fuzzy logic system will be presented. The structure of an IT2FLS, as presented in Fig. 2, is quite similar to a type-1 fuzzy logic system (T1FLS). The only difference is that the antecedent and/or consequent sets in an IT2FLS are type-2, so that each rule output set is a type-2. There are five principal parts in an IT2FLS: Fuzzifier, rule base, inference engine, type-reducer and defuzzifier.

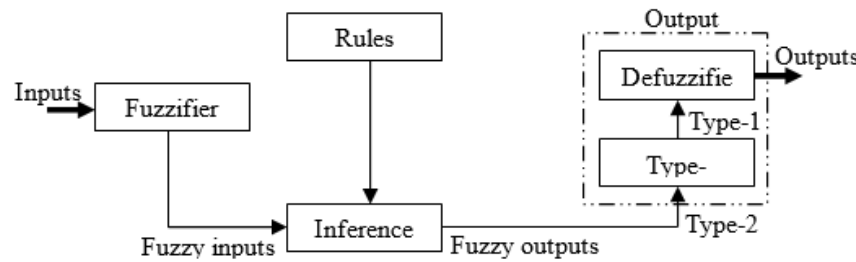

Figure 2. Type-2 fuzzy logic system structure

The type-reducer performs a type-reduction operation which is an extended version of type-1 defuzzification. Type reduction yields a type- 1 set from the type- 2 rule output set. The resulting type-1 set is called type-reduced set. The typereduced set can then be defuzzified to obtain a crisp output. The type reduced set of an IT2FLS shows the possible variation in the crisp output of the fuzzy logic system due to the uncertain natures of the antecedents and/or consequents.

The general form of the $l^{\text {th }}$ rule of the type-2 TakagiSugeno-Kang (TSK) fuzzy logic system can be written as:

If $x_{1}$ is $\widetilde{F}_{1}^{l}$ and $x_{2}$ is $\tilde{F}_{2}^{l}$ and $\ldots x_{\mathrm{n}}$ is $\widetilde{F}_{n}^{l}$, Than

$y^{l}=\tilde{G}^{l} \quad l=1, \ldots, M$ 
where: $\tilde{F}_{j}^{l}$ represent the type- 2 fuzzy system of the input state $j$ of the $l^{\text {th }}$ rule, $x_{1}, x_{2}, \ldots, x_{n}$ are the inputs, $\tilde{G}^{l}$ is the output of type-2 fuzzy system for the rule $l$, and $M$ is the number of rules. As can be seen, the rule structure of type-2 fuzzy logic system is similar to type-1 fuzzy logic system except that type1 membership functions are replaced with their type-2 counterparts.

In Fig. 3, the footprint of uncertainty of each membership function (MF) can be represented as a bounded interval in terms of the upper MF $\bar{\mu}_{\tilde{F}_{j}^{i}}\left(x_{j}\right)$ and the lower $\underline{-}_{\tilde{F}_{j}^{i}}\left(x_{j}\right)$, where

$$
\begin{aligned}
& \bar{\mu}_{\tilde{F}_{j}^{i}}\left(x_{j}\right)=\exp \left[-\frac{1}{2}\left(\frac{x_{j}-m_{j}}{\sigma_{j}}\right)\right] \text { and } \\
& \underline{\mu}_{\tilde{F}_{j}^{i}}\left(x_{j}\right)=0.8 \bar{\mu}_{\tilde{F}_{j}^{i}}\left(x_{j}\right)
\end{aligned}
$$

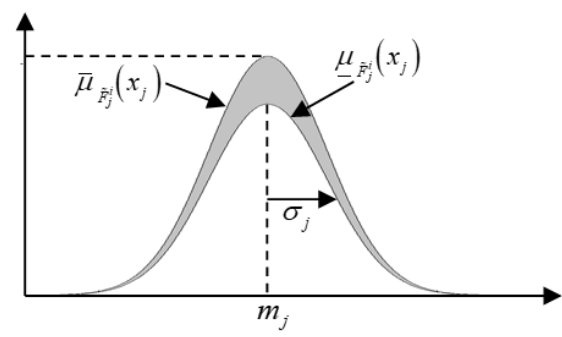

Figure 3. Interval type-2 gaussian MFs

In fuzzy system interval type- 2 using the minimum or product t-norms operations, the $l^{\text {th }}$ activated rule $F^{i}\left(x_{1}, \ldots x_{n}\right)$ gives us the interval that is determined by two extremes $\underline{f}^{l}\left(x_{1}, \ldots x_{n}\right)$ and $\bar{f}^{l}\left(x_{1}, \ldots x_{n}\right)[9]$ :

$$
F^{l}\left(x_{1}, \ldots x_{n}\right)=\left[\underline{f}^{l}\left(x_{1}, \ldots x_{n}\right), \bar{f}^{l}\left(x_{1}, \ldots x_{n}\right)\right] \equiv\left[\underline{f}^{l}, \bar{f}^{l}\right]
$$

with $\underline{f}^{l}$ and $\bar{f}^{l}$ are given as:

$$
\begin{aligned}
& \underline{f}^{l}=\underline{\mu}_{F_{1}^{i}}\left(x_{1}\right) * \ldots * \underline{\mu}_{F_{n}^{i}}\left(x_{n}\right) \\
& \bar{f}^{l}=\bar{\mu}_{F_{1}^{i}}\left(x_{1}\right) * \ldots * \bar{\mu}_{F_{n}^{i}}\left(x_{n}\right)
\end{aligned}
$$

There are many kinds of type-reduction, such as centroid, height, modified weight and center-of-sets [10]. The center-tosets type-reduction will be used in this paper and can be expressed as:

$Y=\left[y_{L}, y_{R}\right]=\int_{y^{1}} \ldots \int_{y^{M}} \ldots \int_{f^{1}} \ldots \int_{f^{M}} 1 / \frac{\sum_{l=1}^{M} f^{l} y^{l}}{\sum_{l=1}^{M} f^{l}}$

Also, $y^{l} \in Y^{l}$ and $Y^{l}=\left[y_{L}^{l}, y_{R}^{l}\right]$ is the centroid of the type2 interval consequent set $\tilde{G}^{l}$, the centroid of a type-2 fuzzy set $[9,10]$. For any value $y \in Y, y$ can be expressed as:

$$
y=\frac{\sum_{l=1}^{M} f^{l} y^{l}}{\sum_{l=1}^{M} f^{l}}
$$

where $y$ is a monotonic increasing function with respect to $y^{l}$. Also, $y_{L}$ is the minimum associated only with $y_{L}^{l}$ and $y_{R}$ is the maximum associated only with $y_{R}^{l}$. Note that $y_{L}$ and $y_{R}$ depend only on the mixture off $\underline{f}^{l}$ or $\bar{f}^{l}$ values. Therefore, the left-most point $y_{L}$ and the right-most point $y_{R}$ can be expressed as a fuzzy basis function (FBF) expansion, i.e.,

$y_{L}=\frac{\sum_{l=1}^{M} f_{L}^{l} y_{L}^{l}}{\sum_{l=1}^{M} f_{L}^{l}}=\theta_{L}^{T} \xi_{L}$

where $\theta_{L}^{l}=y_{L}^{l}$ and $\theta_{L}=\left[\theta_{L}^{1}, \ldots, \theta_{L}^{M}\right]^{T}, \xi_{L}^{l}=\frac{f_{L}^{l}}{\sum_{l=1}^{M} f_{L}^{l}}$ and $\xi_{L}=$ $\left[\xi_{L}^{1}, \ldots, \xi_{L}^{M}\right]^{T}$

$y_{R}=\frac{\sum_{l=1}^{M} f_{R}^{l} y_{R}^{l}}{\sum_{l=1}^{M} f_{R}^{l}}=\theta_{R}^{T} \xi_{R}$

where $\theta_{R}^{l}=y_{R}^{l}$ and $\theta_{R}=\left[\theta_{R}^{1}, \ldots, \theta_{R}^{M}\right]^{T}, \quad \xi_{R}^{l}=\frac{f_{R}^{l}}{\sum_{l=1}^{M} f_{R}^{l}}$ and $\xi_{R}=\left[\xi_{R}^{1}, \ldots, \xi_{R}^{M}\right]^{T}$.

In order to compute $\mathrm{Y}$, we need to compute $y_{L}$ and $y_{R}$. This can be achieved by the use of the iterative Karnik Mendel algorithms procedure given in $[11,12]$.

Initially we compute $y_{L}$

Without loss of generality, assume that $\theta_{L}^{l}$ are arranged in ascending order; i.e., $\theta_{L}^{1} \leq \theta_{L}^{2} \leq \cdots \leq \theta_{L}^{M}$

1. Compute $y_{L}$ in (26) by initially setting $f_{L}^{l}=\frac{f^{l}+\bar{f}^{l}}{2}$ for $l=l, \ldots, M$ where $f^{l}$ and $\bar{f}^{l}$ are given in (23).

2. Find $k(1 \leq \kappa \leq \bar{M}-1)$ such that $\theta_{L}^{\kappa} \leq y_{L} \leq \theta_{L}^{\kappa+1}$.

3. Compute $y_{L}^{\prime}$ in (26) with $f_{L}^{l}=\bar{f}^{l}$ for $l \leq \kappa$ and $f_{L}^{l}=$ $f^{l}$ for $\kappa<l$.

4. If $y_{L}^{\prime} \neq y_{L}$ set $y_{L}=y_{L}^{\prime}$ and go to step 2. If $y_{L}^{\prime}=y_{L}$ then stop and $y_{L}=y_{L}^{\prime}$.

The procedure to compute $y_{R}$ is similar to compute $y_{L}$ in step 2 , it only determines $\kappa(1 \leq \kappa \leq M-1)$, such that $\theta_{L}^{\kappa} \leq$ $y_{R} \leq \theta_{L}^{\kappa+1}$. In step3, compute $y_{R}^{\prime}$ in (27) by $\mathrm{n} f_{R}^{l}=f_{-}^{l}$ for $l \leq$ $\kappa$ and $f_{R}^{l}=\bar{f}^{l}$ for $\kappa<l$. $f^{l}$.

The type-2 fuzzy system output is obtained using the average value of $y_{R}$ and $y_{L}$ given by:

$y=\frac{y_{L}+y_{R}}{2}=\frac{1}{2}\left(\theta_{L}^{T} \xi_{L}+\theta_{R}^{T} \xi_{R}\right)$

\section{ADAPTIVE CONTROLLER DESIGN USING TYPE-1 AND INTERVAL TYPE-2 FUZZY LOGIC SYSTEMS}

The IT2FLS is principally used to estimate the online nonlinear functions $F_{i}\left(\bar{x}_{i}\right)$ and $\bar{g}_{i}\left(\bar{x}_{i}\right)$. These functions are expressed as

$$
\hat{F}_{i}\left(\bar{x}_{i} \backslash \theta_{F_{i}}\right)=\frac{1}{2}\left(\theta_{F_{i} L i}^{T} \xi_{F_{i} L i}+\theta_{F_{i} R i}^{T} \xi_{F_{i} R i}\right)=\theta_{F i}^{T} \xi_{F i}
$$


$\hat{\bar{g}}_{i}\left(\bar{x}_{i} \backslash \theta_{g_{i}}\right)=\frac{1}{2}\left(\theta_{g_{i} L i}^{T} \xi_{g_{i} L i}+\theta_{g_{i} R i}^{T} \xi_{g_{i} R i}\right)=\theta_{g i}^{T} \xi_{g i}$

Using the estimated function (29) and (30), the control laws become:

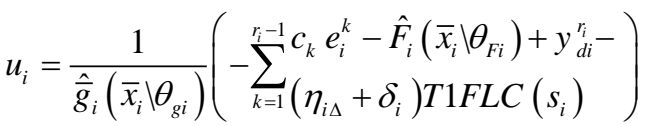

where $\delta_{i}$ is obtained by (2).

\section{Theorem 1.}

Consider the nonlinear system (1) with the control input (31), if the fuzzy-based adaptive laws are chosen as

$$
\begin{aligned}
& \dot{\theta}_{F_{i} L i}=\gamma_{1 i} s_{i} \xi_{F_{i} L i} \\
& \dot{\theta}_{F_{i} R i}=\gamma_{2 i} s_{i} \xi_{F_{i} R i} \\
& \dot{\theta}_{g_{i} L i}=\gamma_{3 i} s_{i} \xi_{g_{i} L i} u_{i} \\
& \dot{\theta}_{g_{i} R i}=\gamma_{4 i} s_{i} \xi_{g_{i} R i} u_{i}
\end{aligned}
$$

where $\gamma_{1 i}, \gamma_{2 i}, \gamma_{3 i}$ and $\gamma_{4 i}$ are positive constants, then, the overall adaptation scheme ensures the overall stability of the closed-loop system resulting in the sense that the tracking error converges to zero asymptotically and all the variables of the closed loop system are bounded.

The optimal parameter estimations of type-2 fuzzy parameters $\theta_{F i}^{*}$ and $\theta_{g i}^{*}$ are defined as:

$$
\begin{aligned}
& \theta_{F i}^{*}=\underset{\theta_{F i} \in \Omega_{F i}}{\arg \min }\left[\sup _{\bar{x}_{i} \in \Re^{n}}\left|\hat{F}_{i}\left(\bar{x}_{i} \backslash \theta_{F i}\right)-F_{i}\left(\bar{x}_{i}\right)\right|\right] \\
& \theta_{\bar{g} i}^{*}=\underset{\theta_{\overline{g i}} \in \Omega_{\overline{\bar{g}}}}{\arg \min }\left[\sup _{\bar{x}_{i} \in \Re^{n}}\left|\hat{\bar{g}}_{i}\left(\bar{x}_{i} \backslash \theta_{g}\right)-\bar{g}_{i}\left(\bar{x}_{i}\right)\right|\right]
\end{aligned}
$$

where $\Omega_{F i}$ and $\Omega_{\bar{g} i}$ are constant sets for $\theta_{F i}$ and $\theta_{\bar{g} i}$ respectively, and they are defined as $\Omega_{F i}=\left\{\theta_{F i} \in \mathfrak{R}^{n}|| \theta_{F i} \mid \leq\right.$ $\left.M_{F_{i}}\right\}$ and $\Omega_{\bar{g} i}=\left\{\theta_{\bar{g} i} \in \mathfrak{R}^{n}|0<| \theta_{\bar{g} i} \mid \leq M_{\bar{g} i}\right\}$, where $M_{F_{i}}$ and $M_{\bar{g} i}$ are positive constant.

Let us define the minimum approximation error:

$\omega_{i}=\left[F_{i}\left(\bar{x}_{i}\right)-\hat{F}_{i}\left(\bar{x}_{i} \backslash \theta_{F i}^{*}\right)\right]+\left[\bar{g}_{i}\left(\bar{x}_{i}\right)-\hat{\bar{g}}_{i}\left(\bar{x}_{i} \backslash \theta_{g i}^{*}\right)\right] u_{i}$

Then from substituting (31) and (38) into (17), the time derivative of sliding surface is obtained by:

$$
\begin{aligned}
& \dot{s}_{i}=F_{i}\left(\bar{x}_{i}\right)-\hat{F}_{i}\left(\bar{x}_{i} \backslash \theta_{F i}\right)+\left(\bar{g}_{i}\left(\bar{x}_{i}\right)-\hat{\bar{g}}_{i}\left(\bar{x}_{i} \backslash \theta_{g i}\right)\right) u_{i} \\
& +\Delta_{i}(t)-\left(\eta_{i \Delta}+\delta_{i}\right) T 1 F L C\left(s_{i}\right) \\
& =\hat{F}_{i}\left(\bar{x}_{i} \backslash \theta_{F i}^{*}\right)-\hat{F}_{i}\left(\bar{x}_{i} \backslash \theta_{F i}\right)+\left(\hat{\bar{g}}_{i}\left(\bar{x}_{i} \backslash \theta_{g i}^{*}\right)-\hat{\bar{g}}_{i}\left(\bar{x}_{i} \backslash \theta_{g i}\right)\right) u_{i} \\
& +\Delta_{i}(t)-\left(\eta_{i \Delta}+\delta_{i}\right) T 1 F L C\left(s_{i}\right)+\omega_{i} \\
& =\left(\theta_{F i}^{* T} \xi_{F i}-\theta_{F i}^{T} \xi_{F i}\right)+\left(\theta_{g i}^{* T} \xi_{g i}-\theta_{g i}^{T} \xi_{g i}\right) u_{i} \\
& +\Delta_{i}(t)-\left(\eta_{i \Delta}+\delta_{i}\right) T 1 F L C\left(s_{i}\right)+\omega_{i}
\end{aligned}
$$

$=\Gamma_{F i}^{T} \xi_{F i}+\Gamma_{g i}^{T} \xi_{g i} u_{i}+\Delta_{i}(t)-\left(\eta_{i \Delta}+\delta_{i}\right) T 1 F L C\left(s_{i}\right)+\omega_{i}$

where: $\Gamma_{F i}=\theta_{F i}^{*}-\theta_{F i}$ and $\Gamma_{g i}=\theta_{g i}^{*}-\theta_{g i}$

The equation (39) is rewritten by:

$\dot{s}_{i}=\frac{1}{2}\left(\Gamma_{F_{i} L i}^{T} \xi_{F_{i} L i}+\Gamma_{F_{i} R i}^{T} \xi_{F_{i} R i}\right)+$

$\frac{1}{2}\left(\Gamma_{g_{i} L i}^{T} \xi_{g_{i} L i}+\Gamma_{g_{i} R i}^{T} \xi_{g_{i} R i}\right) u_{i}$

$+\Delta_{i}(t)-\left(\eta_{i \Delta}+\delta_{i}\right) T 1 F L C\left(s_{i}\right)+\omega_{i}$

\section{Stability analysis}

Let us choose the Lyapunov function candidate as:

$V_{i}=\frac{1}{2} s_{i}^{2}+\frac{1}{4 \gamma_{1 i}} \Gamma_{F_{i} L i}^{T} \Gamma_{F_{i} L i}+\frac{1}{4 \gamma_{2 i}} \Gamma_{F_{i} R i}^{T} \Gamma_{F_{i} R i}$

$+\frac{1}{4 \gamma_{3 i}} \Gamma_{g_{i} L i}^{T} \Gamma_{g_{i} L i}+\frac{1}{4 \gamma_{4 i}} \Gamma_{g_{i} R i}^{T} \Gamma_{g_{i} R i}$

The derivative of (41) with respect to time, gives:

$$
\begin{aligned}
\dot{V}_{i}= & s_{i} \dot{s}_{i}+\frac{1}{2 \gamma_{1 i}} \Gamma_{F_{i} L i}^{T} \dot{\Gamma}_{F_{i} L i}+\frac{1}{2 \gamma_{2 i}} \Gamma_{F_{i} R i}^{T} \dot{\Gamma}_{F_{i} R i} \\
+ & \frac{1}{2 \gamma_{3 i}} \Gamma_{g_{i} L i}^{T} \dot{\Gamma}_{g_{i} L i}+\frac{1}{2 \gamma_{4 i}} \Gamma_{g_{i} R i}^{T} \dot{\Gamma}_{g_{i} R i} \\
& {\left[\frac{1}{2}\left(\Gamma_{F_{i} L i}^{T} \xi_{F_{i} L i}+\Gamma_{F_{i} R i}^{T} \xi_{F_{i} R i}\right)+\right.} \\
= & \left.s_{i} \frac{1}{2}\left(\Gamma_{g_{i} L i}^{T} \xi_{g_{i} L i}+\Gamma_{g_{i} R i}^{T} \xi_{g_{i} R i}\right) u_{i}+\Delta_{i}(t)\right]+ \\
& \left.\frac{1}{2 \gamma_{1 i}} \Gamma_{F_{i} L i}^{T} \dot{\Gamma}_{F_{i} L i}+\frac{1}{2 \gamma_{2 i}} \Gamma_{F_{i} R i}^{T} \dot{\Gamma}_{F_{i} R i}+\delta_{i}\right) T 1 F L C\left(s_{i}\right)+\omega_{i} \\
& \frac{1}{2 \gamma_{3 i}} \Gamma_{g_{i} L i}^{T} \dot{\Gamma}_{g_{i} L i}+\frac{1}{2 \gamma_{4 i}} \Gamma_{g_{i} R i}^{T} \dot{\Gamma}_{g_{i} R i} \\
& =\frac{1}{2 \gamma_{1 i}} \Gamma_{F_{i} L i}^{T}\left(\dot{\Gamma}_{F_{i} L i}+\gamma_{1 i} s_{i} \xi_{F_{i} L i}\right)+ \\
& \frac{1}{2 \gamma_{2 i}} \Gamma_{F_{i} R i}^{T}\left(\dot{\Gamma}_{F_{i} R i}+\gamma_{2 i} s_{i} \xi_{F_{i} R i}\right)+ \\
& \frac{1}{2 \gamma_{3 i}} \Gamma_{g_{i} L i}^{T}\left(\dot{\Gamma}_{g_{i} L i}+\gamma_{3 i} s_{i} \xi_{g_{i} L i} u_{i}\right)+ \\
& \frac{1}{2 \gamma_{4 i}} \Gamma_{g_{i} R i}^{T}\left(\dot{\Gamma}_{g_{i} R i}+\gamma_{4 i} s_{i} \xi_{g_{i} R i} u_{i}\right)+ \\
& s_{i} \Delta_{i}(t)-s_{i}\left(\eta_{i \Delta}+\delta_{i}\right) T 1 F L C\left(s_{i}\right)+s_{i} \omega_{i}
\end{aligned}
$$

where:

$$
\left\{\begin{array}{l}
\dot{\Gamma}_{F_{i} L i}=-\gamma_{1 i} s_{i} \xi_{F_{i} L i} \\
\dot{\Gamma}_{F_{i} R i}=-\gamma_{2 i} s_{i} \xi_{F_{i} R i} \\
\dot{\Gamma}_{g_{i} L i}=-\gamma_{3 i} s_{i} \xi_{g_{i} L i} u_{i} \\
\dot{\Gamma}_{g_{i} R i}=-\gamma_{4 i} s_{i} \xi_{g_{i} R i} u_{i}
\end{array}\right.
$$




$$
\dot{V}_{i} \leq-\left|s_{i}\right|\left(\eta_{i \Delta}\right)+\mu_{s_{i}}\left|\omega_{i}\right|
$$

$$
\begin{aligned}
\dot{V}_{i} & =s_{i} \Delta_{i}(t)-\left(\eta_{i \Delta}+\delta_{i}\right) T 1 F L C\left(s_{i}\right)+s_{i} \omega_{i} \\
& =s_{i} \Delta_{i}(t)-\left(\eta_{i \Delta}+\delta_{i}\right) \sigma_{i}\left|\alpha_{i 1}-\alpha_{i 3}\right|\left|s_{i}\right|+s_{i} \omega_{i} \\
& \leq-\left|s_{i}\right|\left(\eta_{i \Delta}\right)+s_{i} \omega_{i}
\end{aligned}
$$

Since $\omega$ it the minimum approximation error, (44) is the best result that we can obtain. Therefore, all signals in the system are bounded. Obviously, if $e(0)$ is bounded, then $e(t)$ is also bounded for all $t$.

Since the reference signal $y_{d i}^{r_{i}}$ is bounded, then the system states $\bar{x}_{i}$ is bounded as well.

To complete the proof and establish asymptotic convergence of the tracking error, we need proving that $s_{i} \rightarrow$ 0 as $t \rightarrow \infty$ Besides, assume that $\left\|s_{i}\right\| \leq \mu_{s_{i}}$, then Eq. (44) can be rewritten as :
The integral of Eq. (45) provides,

$$
\int_{0}^{T}\left|s_{i}\right| d \tau \leq \frac{1}{\eta_{i \Delta}}\left(\left|V_{i}(0)\right|+\left|V_{i}(T)\right|\right)+\frac{\mu_{s_{i}}}{\eta_{i \Delta}} \int_{0}^{T}\left|\omega_{i}\right| d \tau
$$

The above inequalities mean that all the signals are uniformly bounded in the closed-loop. Then we have $s_{i} \in L_{1}$. Form Eq. (44), we know that $s_{i}$ is bounded and every term in Eq. (44) is bounded. Hence, $\left(s_{i}, \dot{s}_{i}\right) \in L_{\infty}$. We have $s_{i} \rightarrow 0$ as $t \rightarrow \infty$, and from Eq. (4) the tracking error $e_{i}(t)$ will converge to zero, which proves the system stability. The block diagram of the proposed adaptive interval type- 2 fuzzy control system is presented in Fig.4.

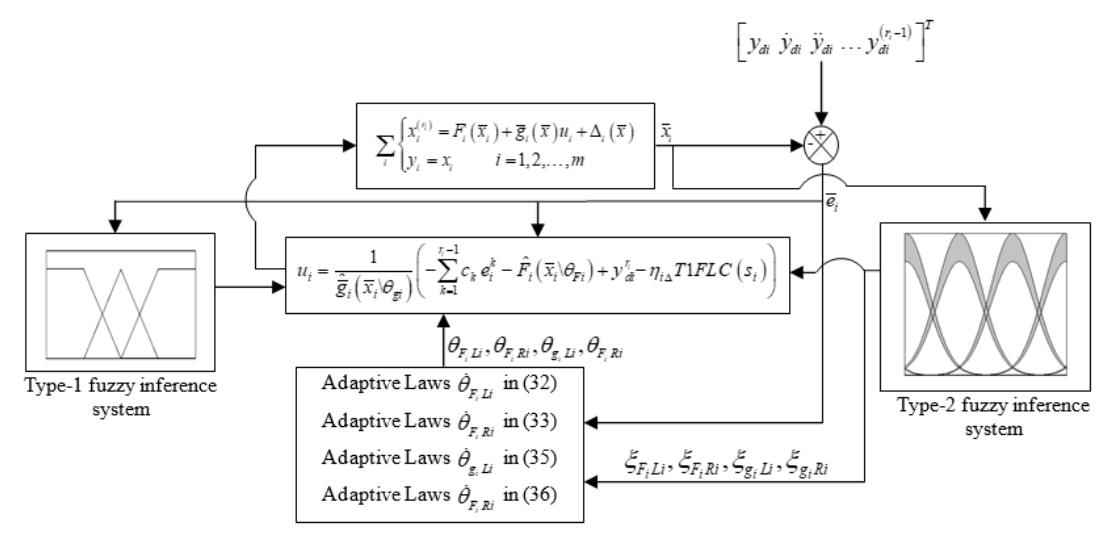

Figure 4. Block diagram of the proposed adaptive interval type-2 fuzzy control system

\section{APPLICATION TO THE COAXIAL OCTOROTOR UAV}

\subsection{Dynamical modeling of the coaxial octorotor}

The configuration of the octorotor is represented in Fig. 5. It is similar to a quadrotor with two coaxial counter-rotating motors at the ends of each arm. This configuration has several advantages compared to the star configuration used in the literature [13] in terms of stability and size. A classical star octorotor needs more arms, and each arm needs to be longer to guarantee adequate spacing among the rotors. We have adopted this configuration for its higher thrust to weight ratio.

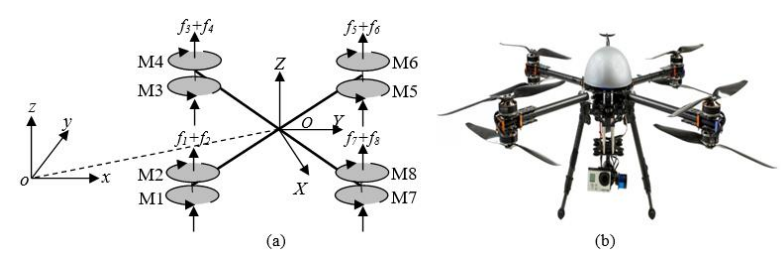

Figure 5. Coaxial octorotor configuration

Consider first a body-fixed reference frame $R_{B}$ with the X, $\mathrm{Y}, \mathrm{Z}$ axis originating at the center of mass of the vehicle.

The $X$ axis points to the front direction, the $Y$ axis to the left direction and the $\mathrm{Z}$ axis upwards. Consider second an inertial frame $R_{I}$ fixed to the earth $\{o, x, y, z\}$.
The equations governing the motion of the system are obtained using the Euler-Lagrange approach and give the commonly used model [14]

$$
\left\{\begin{array}{l}
\ddot{x}=a_{1} \dot{x}+b_{1} u_{1}+W_{1} \\
\ddot{y}=a_{2} \dot{y}+b_{2} u_{2}+W_{2} \\
\ddot{z}=a_{3} \dot{z}+b_{3} u_{3}-g+W_{3} \\
\ddot{\varphi}=a_{4} \dot{\Theta} \dot{\psi}+a_{5} \dot{\varphi}^{2}+a_{6} \dot{\Theta}+b_{4} u_{4}+d_{1} \\
\ddot{\Theta}=a_{7} \dot{\varphi} \dot{\psi}+a_{8} \dot{\Theta}^{2}+a_{9} \dot{\varphi}+b_{5} u_{5}+d_{2} \\
\ddot{\psi}=a_{10} \dot{\varphi} \dot{\Theta}+a_{11} \dot{\psi}^{2}+b_{6} u_{6}+d_{3}
\end{array}\right.
$$

where:

$$
\left\{\begin{array}{l}
a_{1}=\frac{-K_{1}}{m}, a_{2}=\frac{-K_{2}}{m}, a_{3}=\frac{-K_{3}}{m}, \\
a_{4}=\left(\frac{I_{y}-I_{z}}{I_{x}}\right), a_{5}=\frac{-K_{4}}{I_{x}}, a_{6}=\frac{-J_{H} \bar{\Omega}}{I_{x}} \\
a_{7}=\left(\frac{I_{z}-I_{x}}{I_{y}}\right), a_{8}=\frac{-K_{5}}{I_{y}}, a_{9}=\frac{-J_{H} \bar{\Omega}}{I_{y}} \\
a_{10}=\left(\frac{I_{x}-I_{y}}{I_{z}}\right), a_{11}=\frac{-K_{6}}{I_{z}} \\
b_{1}=\frac{u_{3}}{m}, b_{2}=\frac{u_{3}}{m}, b_{3}=\frac{\cos \varphi \cos \Theta}{m}, \\
b_{4}=\frac{1}{I_{x}}, b_{5}=\frac{1}{I_{y}}, b_{6}=\frac{1}{I_{z}}
\end{array}\right.
$$


$\left\{\begin{array}{l}u_{1}=\cos \varphi \sin \Theta \cos \psi+\sin \varphi \sin \psi \\ u_{2}=\cos \varphi \sin \Theta \sin \psi-\sin \varphi \cos \psi\end{array}\right.$

where $m$ denotes the total mass, $g$ represents the acceleration of gravity, $l$ denotes the distance from the center of each rotor to the center of gravity, $K_{1}, \ldots, K_{6}$ denote the drag coefficients and positive constants, $W_{1}, \ldots, W_{3}$ represent the effect of the wind guests on the octorotor translational acceleration, aerodynamics and drag effect and other disturbances, $d_{1}, \ldots, d_{3}$ are the disturbances and drag effects.

$\bar{\Omega}=\Omega_{2}+\Omega_{3}+\Omega_{6}+\Omega_{7}-\Omega_{1}-\Omega_{4}-\Omega_{5}-\Omega_{8}$

$\Omega_{j}$; stand for the angular speed of the propeller $j$ with $j=$ $1, \ldots, 8$.

$I_{x}, I_{y}, I_{z}$ represent the inertias of the coaxial octorotor; $J_{H}$ denotes the inertia of the propeller; $u_{3}$ denotes the total thrust on the body in the z-axis; $u_{4}$ and $u_{5}$ represent the roll and pitch inputs, respectively; $u_{6}$ denotes a yawing moment.

$\left\{\begin{array}{l}u_{3}=F_{1}+F_{2}+F_{3}+F_{4}+F_{5}+F_{6}+F_{7}+F_{8} \\ u_{4}=l \frac{\sqrt{2}}{2}\left(F_{7}+F_{8}+F_{5}+F_{6}-F_{3}-F_{4}-F_{1}-F_{2}\right) \\ u_{5}=l \frac{\sqrt{2}}{2}\left(F_{3}+F_{4}+F_{5}+F_{6}-F_{7}-F_{8}-F_{1}-F_{2}\right) \\ u_{6}=\frac{d}{b}\left(F_{2}+F_{3}+F_{6}+F_{7}-F_{1}-F_{4}-F_{5}-F_{8}\right)\end{array}\right.$

where $F_{j}=b \Omega_{j}^{2}$ with $j=1, \ldots, 8$ denote the thrust generated by eight rotors and are considered as the real control inputs to the dynamical system, $b$ denotes the lift coefficient; $d$ denotes the force to moment scaling factor.

\section{Assumption 1}

The roll, the pitch and the yaw angles $(\varphi, \theta, \psi)$ are bounded as follows: roll angle by $\frac{-\pi}{2}<\varphi<\frac{\pi}{2}$; pitch angle, $\frac{-\pi}{2}<\Theta<\frac{\pi}{2}$; and yaw angle, $-\pi<\psi<\pi$.

The dynamic model (47) can be rewritten as follows

$$
\begin{aligned}
& \sum_{1}\left\{\begin{array}{l}
\ddot{x}=F_{1}(x, \dot{x})+\bar{g}_{1} u_{1}+\Delta_{1} \\
y_{1}=x
\end{array}\right. \\
& \sum_{2}\left\{\begin{array}{l}
\ddot{y}=F_{2}(y, \dot{y})+\bar{g}_{2} u_{2}+\Delta_{2} \\
y_{2}=y
\end{array}\right. \\
& \sum_{3}\left\{\begin{array}{l}
\ddot{z}=F_{3}(z, \dot{z})+\bar{g}_{3} u_{3}+\Delta_{3} \\
y_{3}=z
\end{array}\right. \\
& \sum_{4}\left\{\begin{array}{l}
\ddot{\varphi}=F_{4}(\varphi, \dot{\varphi})+\bar{g}_{4} u_{4}+\Delta_{4} \\
y_{4}=\varphi
\end{array}\right. \\
& \sum_{5}\left\{\begin{array}{l}
\ddot{\Theta}=F_{5}(\Theta, \dot{\Theta})+\bar{g}_{5} u_{5}+\Delta_{5} \\
y_{5}=\Theta
\end{array}\right. \\
& \sum_{6}\left\{\begin{array}{l}
\ddot{\psi}=F_{5}(\psi, \dot{\psi})+\bar{g}_{6} u_{6}+\Delta_{6} \\
y_{6}=\psi
\end{array}\right.
\end{aligned}
$$

where:

$$
\left\{\begin{array} { l } 
{ F _ { 1 } ( x , \dot { x } ) = a _ { 1 } \dot { x } } \\
{ F _ { 2 } ( y , \dot { y } ) = a _ { 2 } \dot { y } } \\
{ F _ { 3 } ( z , \dot { z } ) = a _ { 3 } \dot { z } - g } \\
{ F _ { 4 } ( \varphi , \dot { \varphi } ) = a _ { 5 } \dot { \varphi } ^ { 2 } } \\
{ F _ { 5 } ( \Theta , \dot { \Theta } ) = a _ { 8 } \dot { \Theta } ^ { 2 } } \\
{ F _ { 6 } ( \psi , \dot { \psi } ) = a _ { 1 1 } \dot { \psi } ^ { 2 } }
\end{array} \left\{\begin{array} { l } 
{ \overline { g } _ { 1 } = b _ { 1 } } \\
{ \overline { g } _ { 2 } = b _ { 2 } } \\
{ \overline { g } _ { 3 } = b _ { 3 } } \\
{ \overline { g } _ { 4 } = b _ { 4 } } \\
{ \overline { g } _ { 5 } = b _ { 5 } } \\
{ \overline { g } _ { 6 } = b _ { 6 } }
\end{array} \quad \left\{\begin{array}{l}
\Delta_{1}=W_{1} \\
\Delta_{2}=W_{2} \\
\Delta_{3}=W_{3} \\
\Delta_{4}=d_{1} \\
\Delta_{5}=d_{2} \\
\Delta_{6}=d_{3}
\end{array}\right.\right.\right.
$$

In model (50) to (55), we have $m=6, r_{i=1, \ldots, 6}=2$, and $x=$ $[x, \dot{x}, y, \dot{y}, z, \dot{z}, \varphi, \dot{\varphi}, \Theta, \dot{\Theta}, \psi, \dot{\psi}]^{T_{112}}{ }^{T}$

\subsection{Control strategy of the coaxial octorotor}

Before synthesizing the control law the following assumption are assumed [14]:

\section{Assumption 2}

The variables $\varphi$ and $\Theta$ move exclusively inside the open set $\left(\frac{-\pi}{2}, \frac{\pi}{2}\right)$ and the thrust $U_{1}$ is always has to be strictly positive $\left(U_{1}>0\right)$; otherwise, the device will certainly fall.

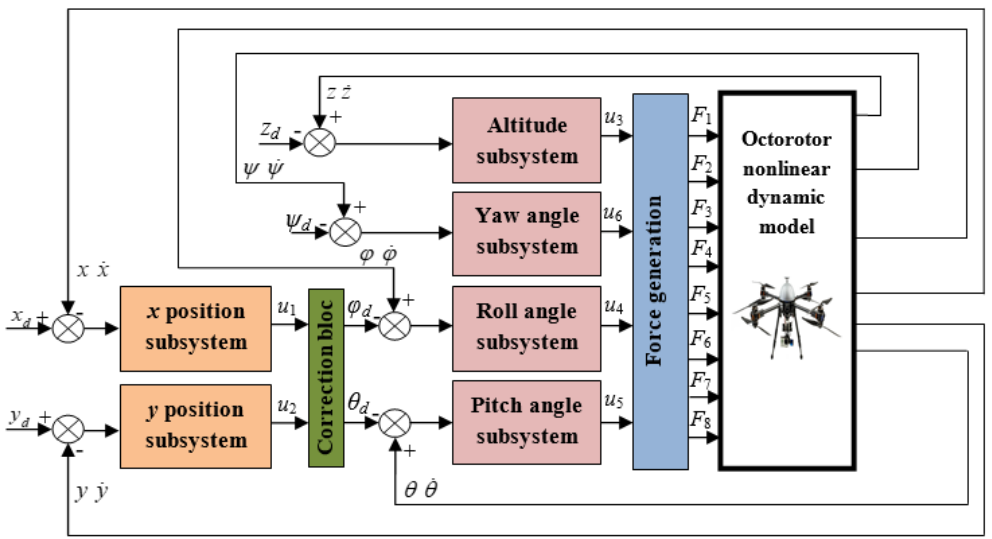

Figure 6. Bloc diagram of the proposed flight control strategy

This assumption reflects a realistic situation. That is, exclude the singular cases where the aircraft is either vertically, horizontally inclined, or is free falling. Therefore, the adopted control strategy cannot be applied to these singular cases [14]. 
The translational motion control is performed in two stages. In the first one, the helicopter height, $z$, is controlled and the total thrust, $u_{3}$, is the manipulated signal. In the second stage, the reference of pitch and roll angles $\left(\Theta_{d}\right.$ and $\varphi_{d}$, respectively) are generated through the two virtual inputs $u_{1}$ and $u_{2}$, computed to follow the desired $x y$ movement. Finally the rotation controller is used to stabilize the octorotor under near quasi-stationary conditions with control inputs $u_{4}, u_{5}, u_{6}$. The overall scheme of the control strategy is depicted in Fig.6.

\subsection{1 x position control}

The $x$ position subsystem is presented by:

$\sum_{1}\left\{\begin{array}{l}\ddot{x}=F_{1}(x, \dot{x})+\bar{g}_{1} u_{1}+\Delta_{1} \\ y_{1}=x\end{array}\right.$

with $\bar{x}_{1}=[x, \dot{x}]$

To apply the proposed control based on an adaptive type-2 fuzzy controller, the functions $F_{1}(x, \dot{x}), \bar{g}_{1}$ have been estimated online by zero-order type-2 Takagi-Sugeno fuzzy systems. The functions are estimated by:

$$
\begin{aligned}
& \hat{F}_{1}\left(\bar{x}_{1} \backslash \theta_{F_{1}}\right)=\frac{1}{2}\left(\theta_{F_{1} L_{1}}^{T} \xi_{F_{1} L_{1}}+\theta_{F_{1} R_{1}}^{T} \xi_{F_{1} R_{1}}\right) \\
& \hat{\bar{g}}_{1}\left(\bar{x}_{1} \backslash \theta_{g_{1}}\right)=\frac{1}{2}\left(\theta_{g_{1} L_{1}}^{T} \xi_{g_{1} L_{1}}+\theta_{g_{1} R_{1}}^{T} \xi_{g_{1} R_{1}}\right)
\end{aligned}
$$

where $\theta_{F_{1} L_{1}}^{T}, \theta_{F_{1} R_{1}}^{T}, \theta_{g_{1} L_{1}}^{T}$ and $\theta_{g_{1} R_{1}}^{T}$ are the vector parameter of the fuzzy system. The fuzzy system has two inputs $x$ and $\dot{x}$, each input is described by seven interval type-2 Gaussian MFs.

The control law that is provided by the adaptive controller is expressed by:

$u_{1}=\frac{1}{\bar{g}_{1}\left(\bar{x}_{1} \backslash \theta_{g 1}\right)}\left(-c_{1} \dot{e}_{1}-\hat{F}_{1}\left(\bar{x}_{1} \backslash \theta_{F 1}\right)+\ddot{y}_{d 1}-\eta_{1 \Delta} T 1 F L C\left(s_{1}\right)\right)$

where: $e_{1}=x-x_{d}, \dot{e}_{1}=\dot{x}-\dot{x}_{d}, \ddot{y}_{d 1}=\ddot{x}_{d}, s_{1}=\dot{e}_{1}+c_{1} e_{1}$ and $\left(\eta_{1 \Delta}, c_{1}\right)>0$.

The type-2 fuzzy systems parameters are adjusted by the following law:

$$
\left\{\begin{array}{l}
\dot{\theta}_{F_{1} L 1}=\gamma_{11} s_{1} \xi_{F_{1} L 1} \\
\dot{\theta}_{F_{1} R 1}=\gamma_{21} s_{1} \xi_{F_{1} R 1} \\
\dot{\theta}_{g_{1} L 1}=\gamma_{31} s_{1} \xi_{g_{1} L 1} u_{1} \\
\dot{\theta}_{g_{1} R 1}=\gamma_{41} s_{1} \xi_{g_{1} R 1} u_{1}
\end{array}\right.
$$

where $\gamma_{11}, \gamma_{21}, \gamma_{31}$ and $\gamma_{41}$ are the positive constant.

\subsection{2 y position control}

The $y$ position subsystem is presented by:

$\sum_{2}\left\{\begin{array}{l}\ddot{y}=F_{2}(y, \dot{y})+\bar{g}_{2} u_{2}+\Delta_{2} \\ y_{2}=y\end{array}\right.$

With $\bar{x}_{2}=[y, \dot{y}]$
To apply the proposed control based on an adaptive type-2 fuzzy controller, the functions $F_{2}(y, \dot{y}), \bar{g}_{2}$ have been estimated online by zero-order type-2 Takagi-Sugeno fuzzy systems. The functions are estimated by:

$$
\begin{aligned}
& \hat{F}_{2}\left(\bar{x}_{2} \backslash \theta_{F 2}\right)=\frac{1}{2}\left(\theta_{F_{2} L_{2}}^{T} \xi_{F_{2} L_{2}}+\theta_{F_{2} R_{2}}^{T} \xi_{F_{2} R_{2}}\right) \\
& \hat{\bar{g}}_{2}\left(\bar{x}_{2} \backslash \theta_{g_{2}}\right)=\frac{1}{2}\left(\theta_{g_{2} L_{2}}^{T} \xi_{g_{2} L_{2}}+\theta_{g_{2} R_{2}}^{T} \xi_{g_{2} R_{2}}\right)
\end{aligned}
$$

where $\theta_{F_{2} L_{2}}^{T}, \theta_{F_{2} R_{2}}^{T}, \theta_{g_{2} L_{2}}^{T}$ and $\theta_{g_{2} R_{2}}^{T}$ are the vector parameter of the fuzzy system. The fuzzy system has two inputs $y$ and $\dot{y}$, each input is described by seven interval type2 Gaussian MFs.

The control law that is provided by the adaptive controller is expressed by:

$u_{2}=\frac{1}{\bar{g}_{2}\left(\bar{x}_{2} \backslash \theta_{g 2}\right)}\left(\begin{array}{l}-c_{2} \dot{e}_{2}-\hat{F}_{2}\left(\bar{x}_{2} \backslash \theta_{F 2}\right)+ \\ \ddot{y}_{d 2}-\eta_{2 \Delta} \operatorname{T1FLC}\left(s_{2}\right)\end{array}\right)$

where:

$e_{2}=y-y_{d}, \quad \dot{e}_{2}=\dot{y}-\dot{y}_{d}, \quad \ddot{y}_{d 2}=\ddot{y}_{d}, \quad s_{2}=\dot{e}_{2}+c_{2} e_{2}$ and $\left(\eta_{2 \Delta}, c_{2}\right)>0$

The type- 2 fuzzy systems parameters are adjusted by the following law:

$\left\{\begin{array}{l}\dot{\theta}_{F_{2} L 2}=\gamma_{12} s_{2} \xi_{F_{2} L 2} \\ \dot{\theta}_{F_{2} R 2}=\gamma_{22} s_{2} \xi_{F_{2} R 2} \\ \dot{\theta}_{g_{2} L 2}=\gamma_{32} s_{2} \xi_{g_{2} L 2} u_{2} \\ \dot{\theta}_{g_{2} R 2}=\gamma_{42} s_{2} \xi_{g_{2} R 2} u_{2}\end{array}\right.$

where $\gamma_{12}, \gamma_{22}, \gamma_{32}$ and $\gamma_{42}$ are the positive constant.

\subsubsection{Altitude control (z)}

The $z$ position subsystem is presented by:

$\sum_{3}\left\{\begin{array}{l}\ddot{z}=F_{3}(z, \dot{z})+\bar{g}_{3} u_{3}+\Delta_{3} \\ y_{3}=z\end{array}\right.$

With $\bar{x}_{3}=[z, \dot{z}]$

To apply the proposed control based on an adaptive type-2 fuzzy controller, the functions $F_{3}(z, \dot{z}), \bar{g}_{3}$ have been estimated online by zero-order type-2 Takagi-Sugeno fuzzy systems. The functions are estimated by:

$$
\begin{aligned}
& \hat{F}_{3}\left(\bar{x}_{3} \backslash \theta_{F 3}\right)=\frac{1}{2}\left(\theta_{F_{3} L_{3}}^{T} \xi_{F_{3} L_{3}}+\theta_{F_{3} R_{3}}^{T} \xi_{F_{3} R_{3}}\right) \\
& \hat{\bar{g}}_{3}\left(\bar{x}_{3} \backslash \theta_{g_{3}}\right)=\frac{1}{2}\left(\theta_{g_{3} L_{3}}^{T} \xi_{g_{3} L_{3}}+\theta_{g_{3} R_{3}}^{T} \xi_{g_{3} R_{3}}\right)
\end{aligned}
$$

where $\theta_{F_{3} L_{3}}^{T}, \theta_{F_{3} R_{3}}^{T}, \theta_{g_{3} L_{3}}^{T}$ and $\theta_{g_{3} R_{3}}^{T}$ are the vector parameter of the fuzzy system. The fuzzy system has two inputs $z$ and $\dot{z}$, each input is described by seven interval type-2 Gaussian MFs. 
The control law that is provided by the adaptive controller is expressed by:

$u_{3}=\frac{1}{\bar{g}_{3}\left(\bar{x}_{3} \backslash \theta_{g 3}\right)}\left(\begin{array}{l}-c_{3} \dot{e}_{3}-\hat{F}_{3}\left(\bar{x}_{3} \backslash \theta_{F 3}\right)+\ddot{y}_{d 3} \\ -\eta_{3 \Delta} T 1 F L C\left(s_{3}\right)\end{array}\right)$

where:

$e_{3}=z-z_{d}, \dot{e}_{3}=\dot{z}-\dot{z}_{d}, \ddot{y}_{d 3}=\ddot{z}_{d}, s_{3}=\dot{e}_{3}+c_{3} e_{3}$ and $\left(\eta_{3_{\Delta}}, c_{3}\right)>0$.

The type- 2 fuzzy systems parameters are adjusted by the following law:

$$
\left\{\begin{array}{l}
\dot{\theta}_{F_{3} L 3}=\gamma_{13} s_{3} \xi_{F_{3} L 3} \\
\dot{\theta}_{F_{3} R 3}=\gamma_{23} s_{3} \xi_{F_{3} R 3} \\
\dot{\theta}_{g_{3} L 3}=\gamma_{33} s_{3} \xi_{g_{3} L 3} u_{3} \\
\dot{\theta}_{g_{3} R 3}=\gamma_{43} s_{3} \xi_{g_{3} R 3} u_{3}
\end{array}\right.
$$

where $\gamma_{13}, \gamma_{23}, \gamma_{33}$ and $\gamma_{43}$ are the positive constant.

\subsubsection{Roll control $(\varphi)$}

The roll angle subsystem is presented by:

$\sum_{4}\left\{\begin{array}{l}\ddot{\varphi}=F_{4}(\varphi, \dot{\varphi})+\bar{g}_{4} u_{4}+\Delta_{4} \\ y_{4}=\varphi\end{array}\right.$

With $\bar{x}_{4}=[\varphi, \dot{\varphi}]$

To apply the proposed control based on an adaptive type-2 fuzzy controller, the functions $F_{4}(\varphi, \dot{\varphi}), \bar{g}_{4}$ have been estimated online by zero-order type-2 Takagi-Sugeno fuzzy systems. The functions are estimated by:

$$
\begin{aligned}
& \hat{F}_{4}\left(\bar{x}_{4} \backslash \theta_{F 4}\right)=\frac{1}{2}\left(\theta_{F_{4} L_{4}}^{T} \xi_{F_{4} L_{4}}+\theta_{F_{4} R_{4}}^{T} \xi_{F_{4} R_{4}}\right) \\
& \hat{\bar{g}}_{4}\left(\bar{x}_{4} \backslash \theta_{g_{4}}\right)=\frac{1}{2}\left(\theta_{g_{4} L_{4}}^{T} \xi_{g_{4} L_{4}}+\theta_{g_{4} R_{4}}^{T} \xi_{g_{4} R_{4}}\right)
\end{aligned}
$$

where $\theta_{F_{4} L_{4}}^{T}, \theta_{F_{4} R_{4}}^{T}, \theta_{g_{4} L_{4}}^{T}$ and $\theta_{g_{4} R_{4}}^{T}$ are the vector parameter of the fuzzy system. The fuzzy system has two inputs $\varphi$ and $\dot{\varphi}$, each input is described by seven interval type-2 Gaussian MFs.

The control law that is provided by the adaptive controller is expressed by:

$u_{4}=\frac{1}{\bar{g}_{4}\left(\bar{x}_{4} \backslash \theta_{g 4}\right)}\left(\begin{array}{l}-c_{4} \dot{e}_{4}-\hat{F}_{4}\left(\bar{x}_{4} \backslash \theta_{F 4}\right)+\ddot{y}_{d 4} \\ -\eta_{4 \Delta} T 1 F L C\left(s_{4}\right)\end{array}\right)$

where: $e_{4}=z-z_{d}, \dot{e}_{4}=\dot{z}-\dot{z}_{d}, \ddot{y}_{d 4}=\ddot{z}_{d}, s_{4}=\dot{e}_{4}+c_{4} e_{4}$ and $\left(\eta_{4_{\Delta}}, c_{4}\right)>0$.

The type-2 fuzzy systems parameters are adjusted by the following law:

$$
\left\{\begin{array}{l}
\dot{\theta}_{F_{4} L 4}=\gamma_{14} s_{4} \xi_{F_{4} L 4} \\
\dot{\theta}_{F_{4} R 4}=\gamma_{24} s_{4} \xi_{F_{4} R 4} \\
\dot{\theta}_{g_{4} L 4}=\gamma_{34} s_{4} \xi_{g_{4} L 4} u_{4} \\
\dot{\theta}_{g_{4} R 4}=\gamma_{44} s_{4} \xi_{g_{4} R 4} u_{4}
\end{array}\right.
$$

where $\gamma_{14}, \gamma_{24}, \gamma_{34}$ and $\gamma_{44}$ are the positive constant.

The desired roll angel is given by:

$\varphi_{d}=-\operatorname{arctg}\left(u_{2}\right)$

\subsubsection{Pitch control $(\Theta)$}

The pitch angle subsystem is presented by:

$\sum_{5}\left\{\begin{array}{l}\ddot{\Theta}=F_{5}(\Theta, \dot{\Theta})+\bar{g}_{5} u_{5}+\Delta_{5} \\ y_{5}=\Theta\end{array}\right.$

with $\bar{x}_{5}=[\Theta, \dot{\Theta}]$

To apply the proposed control based on an adaptive type-2 fuzzy controller, the functions $F_{5}(\Theta, \dot{\Theta}), \bar{g}_{5}$ have been estimated online by zero-order type-2 Takagi-Sugeno fuzzy systems. The functions are estimated by:

$\hat{F}_{5}\left(\bar{x}_{5} \backslash \theta_{F 5}\right)=\frac{1}{2}\left(\theta_{F_{5} L_{5}}^{T} \xi_{F_{5} L_{5}}+\theta_{F_{5} R_{5}}^{T} \xi_{F_{5} R_{5}}\right)$

$\hat{\bar{g}}_{5}\left(\bar{x}_{5} \backslash \theta_{g_{5}}\right)=\frac{1}{2}\left(\theta_{g_{5} L_{5}}^{T} \xi_{g_{5} L_{5}}+\theta_{g_{5} R_{5}}^{T} \xi_{g_{5} R_{5}}\right)$

where $\theta_{F_{5} L_{5}}^{T}, \theta_{F_{5} R 5}^{T}, \theta_{g_{5} L_{5}}^{T}$ and $\theta_{g_{5} R_{5}}^{T}$ are the vector parameter of the fuzzy system. The fuzzy system has two inputs $\Theta$ and $\dot{\Theta}$, each input is described by seven interval type-2 Gaussian MFs.

The control law that is provided by the adaptive controller is expressed by:

$u_{5}=\frac{1}{\bar{g}_{5}\left(\bar{x}_{5} \backslash \theta_{g 5}\right)}\left(\begin{array}{l}-c_{5} \dot{e}_{5}-\hat{F}_{5}\left(\bar{x}_{5} \backslash \theta_{F 5}\right)+\ddot{y}_{d 5} \\ -\eta_{5 \Delta} T 1 F L C\left(s_{5}\right)\end{array}\right)$

where: $e_{5}=\Theta-\Theta_{d}, \dot{e}_{5}=\dot{\Theta}-\dot{\Theta}_{d}, \ddot{y}_{d 5}=\ddot{\Theta}_{d}, s_{5}=\dot{e}_{5}+c_{5}$ $e_{5}$ and $\left(\eta_{5}, c_{5}\right)>0$.

The type- 2 fuzzy systems parameters are adjusted by the following law:

$\left\{\begin{array}{l}\dot{\theta}_{F_{5} L 5}=\gamma_{15} s_{5} \xi_{F_{5} L 5} \\ \dot{\theta}_{F_{5} R 5}=\gamma_{25} s_{5} \xi_{F_{5} R 5} \\ \dot{\theta}_{g_{5} L 5}=\gamma_{35} s_{5} \xi_{g_{5} L 5} u_{5} \\ \dot{\theta}_{g_{5} R 5}=\gamma_{45} s_{5} \xi_{g_{5} R 5} u_{5}\end{array}\right.$

where $\gamma_{15}, \gamma_{25}, \gamma_{35}$ and $\gamma_{45}$ are the positive constant.

The desired pitch angel is given by:

$\Theta_{d}=\operatorname{arctg}\left(u_{1} \cos (\varphi)\right)$

5.2.6 Yaw control $(\psi)$

The yaw angle subsystem is presented by:

$\sum_{6}\left\{\begin{array}{l}\ddot{\psi}=F_{5}(\psi, \dot{\psi})+\bar{g}_{6} u_{6}+\Delta_{6} \\ y_{6}=\psi\end{array}\right.$

With $\bar{x}_{6}=[\psi, \dot{\psi}]$ 
To apply the proposed control based on an adaptive type-2 fuzzy controller, the functions $F_{6}(\psi, \dot{\psi}), \bar{g}_{6}$ have been estimated online by zero-order type-2 Takagi-Sugeno fuzzy systems. The functions are estimated by:

$$
\begin{aligned}
& \hat{F}_{6}\left(\bar{x}_{6} \backslash \theta_{F 6}\right)=\frac{1}{2}\left(\theta_{F_{6} L_{6}}^{T} \xi_{F_{6} L_{6}}+\theta_{F_{6} R_{6}}^{T} \xi_{F_{6} R_{6}}\right) \\
& \hat{\bar{g}}_{6}\left(\bar{x}_{6} \backslash \theta_{g_{6}}\right)=\frac{1}{2}\left(\theta_{g_{6} L_{6}}^{T} \xi_{g_{6} L_{6}}+\theta_{g_{6} R_{6}}^{T} \xi_{g_{6} R_{6}}\right)
\end{aligned}
$$

where $\theta_{F_{6} L_{6}}^{T}, \theta_{F_{6} R 6}^{T}, \theta_{g_{6} L_{6}}^{T}$ and $\theta_{g_{6} R 6}^{T}$ are the vector parameter of the fuzzy system. The fuzzy system has two inputs $\psi$ and $\dot{\psi}$, each input is described by seven interval type-2 Gaussian MFs.

The control law that is provided by the adaptive controller is expressed by:

$$
u_{6}=\frac{1}{\bar{g}_{6}\left(\bar{x}_{6} \backslash \theta_{g 6}\right)}\left(\begin{array}{l}
-c_{6} \dot{e}_{6}-\hat{F}_{6}\left(\bar{x}_{6} \backslash \theta_{F 6}\right)+ \\
\ddot{y}_{d 6}-\eta_{6 \Delta} T 1 F L C\left(s_{6}\right)
\end{array}\right)
$$

where: $e_{6}=\psi-\psi_{d}, \dot{e}_{6}=\dot{\psi}-\dot{\psi}_{d}, \ddot{y}_{d 6}=\ddot{\psi}_{d}, s_{6}=\dot{e}_{6}+c_{6}$ $e_{6}$ and $\left(\eta_{6 \Delta}, c_{6}\right)>0$.

The type-2 fuzzy systems parameters are adjusted by the following law:

$$
\left\{\begin{array}{l}
\dot{\theta}_{F_{6} L 6}=\gamma_{16} s_{6} \xi_{F_{6} L 6} \\
\dot{\theta}_{F_{6} R 6}=\gamma_{26} s_{6} \xi_{F_{6} R 6} \\
\dot{\theta}_{g_{6} L 6}=\gamma_{36} s_{6} \xi_{g_{6} L 6} u_{6} \\
\dot{\theta}_{g_{6} R 6}=\gamma_{46} s_{6} \xi_{g_{6} R 6} u_{6}
\end{array}\right.
$$

where $\gamma_{16}, \gamma_{26}, \gamma_{36}$ and $\gamma_{46}$ are the positive constant.

\section{SIMULATION RESULTS}

The proposed control strategy has been tested by simulation in order to check the effectiveness and the performance attained for the path following problem. The nominal parameters of the coaxial octorotor system are given as [14] presented by the following table.

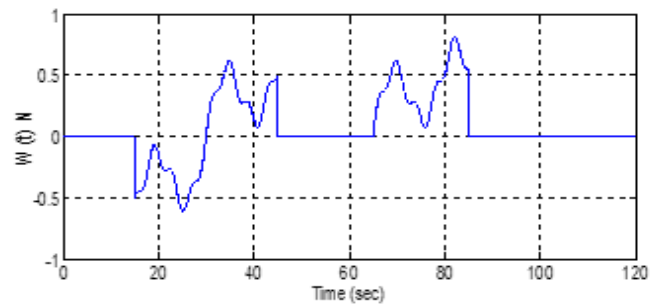

(a)
Table 2. Physical parameters of the coaxial octorotor

\begin{tabular}{|l|l|}
\hline Symbol & Value \\
\hline$I_{x}$ & $4.2 \times 10^{-2}\left(\mathrm{Kg} . \mathrm{m}^{2}\right)$ \\
\hline$I_{y}$ & $4.2 \times 10^{-2}\left(\mathrm{Kg} \cdot \mathrm{m}^{2}\right)$ \\
\hline$I_{Z}$ & $7.5 \times 10^{-2}\left(\mathrm{Kg} \cdot \mathrm{m}^{2}\right)$ \\
\hline$K_{p}$ & $2.9842 \times 10^{-5}\left(\mathrm{Ns}^{2}\right)$ \\
\hline$K_{d}$ & $3.2320 \times 10^{-7}\left(\mathrm{Nms}^{2}\right)$ \\
\hline$m$ & $1.6(\mathrm{Kg})$ \\
\hline $\mathrm{g}$ & $9.81\left(\mathrm{~m} / \mathrm{s}^{2}\right)$ \\
\hline$l$ & $0.23(\mathrm{~m})$ \\
\hline$K_{I}$ & $5.5670 \times 10^{-4}(\mathrm{~N} / \mathrm{m} / \mathrm{s})$ \\
\hline$K_{2}$ & $5.5670 \times 10^{-4}(\mathrm{~N} / \mathrm{m} / \mathrm{s})$ \\
\hline$K_{3}$ & $6.3540 \times 10^{-4}(\mathrm{~N} / \mathrm{m} / \mathrm{s})$ \\
\hline$K_{4}$ & $5.5670 \times 10^{-4}(\mathrm{~N} / \mathrm{rad} / \mathrm{s})$ \\
\hline$K_{5}$ & $5.5670 \times 10^{-4}(\mathrm{~N} / \mathrm{rad} / \mathrm{s})$ \\
\hline$K_{6}$ & $6.3540 \times 10^{-4}(\mathrm{~N} / \mathrm{rad} / \mathrm{s})$ \\
\hline
\end{tabular}

Two flight tests have been carried out to demonstrate the performance of the proposed control. First, an application which does not take into accounts the external disturbances and model uncertainty on the six degrees of freedom. Secondly, a simulation with parametric variations (at $\mathrm{t}=60 \mathrm{~s}$ ). Indeed, these variations impose an increase of $75 \%$ of the inertias $\left(I_{x}\right.$, $I_{y}$ and $I_{z}$ ) and an increase of $50 \%$ on the masse and with the wind and torques disturbances are introduced, which are chosen as:

$$
W_{i}(t)=\left\{\begin{array}{l}
0 N, \quad 0 \leq t \leq 15 \\
0.5 \sin \frac{\pi(t-30)}{31}+0.3 \sin \frac{\pi(t-30)}{7}+ \\
0.08 \sin \frac{\pi(t-30)}{2} \\
+0.056 \sin \frac{\pi(t-30)}{11} N, 15 \leq t \leq 45 \\
0 N, \quad 45 \leq t \leq 65 \\
0.5 \sin \frac{\pi(t-65)}{31}+0.3 \sin \frac{\pi(t-65)}{7} \\
+0.08 \sin \frac{\pi(t-65)}{2} \\
+0.056 \sin \frac{\pi(t-65)}{11} \\
0 N, \quad 85 \leq t \leq 100
\end{array} \quad \text { for } \quad 65 \leq t \leq 85,3\right.
$$

The disturbances $W_{i}(t)$ and $d_{i}(t)$ has been depicted in Fig.7.

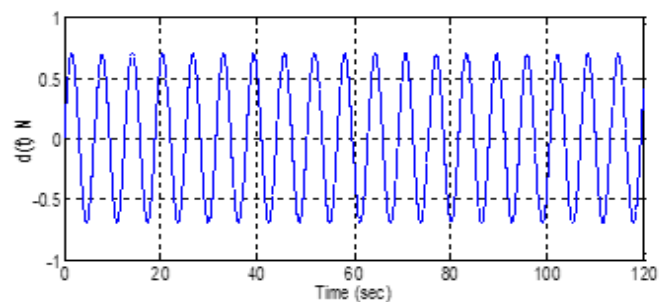

(b)

Figure 7. The disturbances effect. (a) Wind effect. (b) torques disturbances effect 


\subsection{Flight with wind and torques disturbances and uncertainties of the inertia and masse}

To demonstrate the robustness, the wind disturbances are selected like in (88), the torques disturbances are selected like in (107) and the inertias uncertainties are presented in Fig.7. The obtained results are depicted in Figs.8-11.

Fig. 8 shows the absolute position of the octorotor during its flight, when the wind force, torques disturbances and parametric variations are introduced, the assigned navigational task are successfully achieved and the reference trajectories
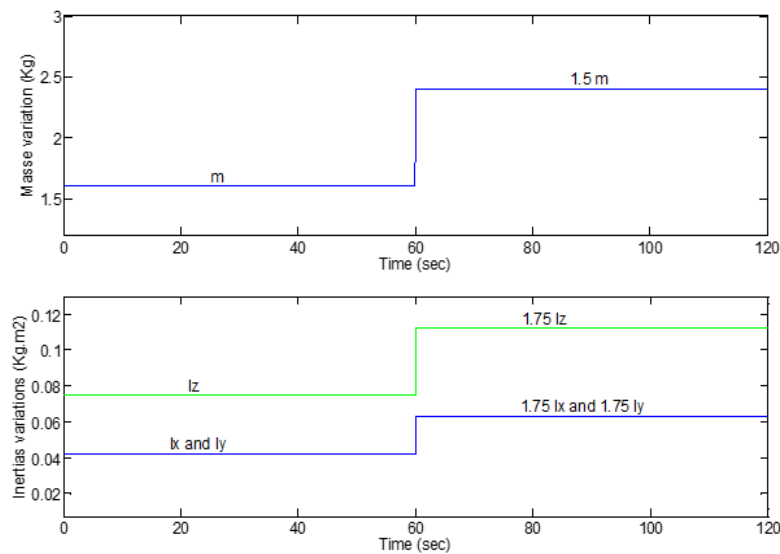

Figure 7. Evolution of the inertias and masse variation
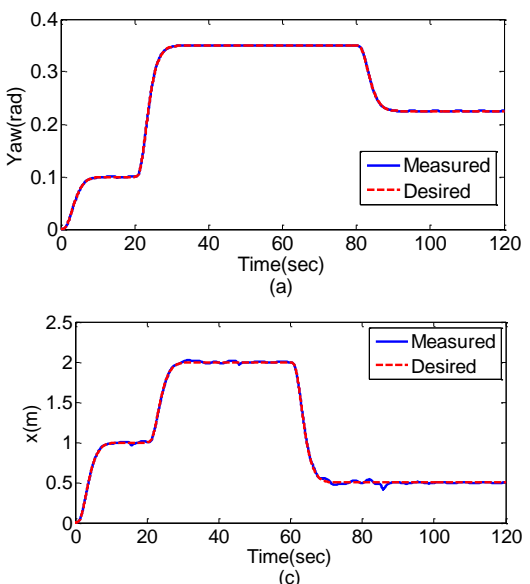

are tracked with high accuracy. Fig.9 represents the position trajectories. From these figures, we can see a well good tracking of the desired trajectories, the controller is able to reject them showing the robustness of the proposed control approach and the stability of the closed loop dynamics is guaranteed, we can notice in Fig.10 an optimization of tilt angles (pitch and roll) and consequently the use of minimal energy. On the other hand, it can be seen from the control curvature presented by Fig. 11 a smooth signal could easily be applied to a real life model.

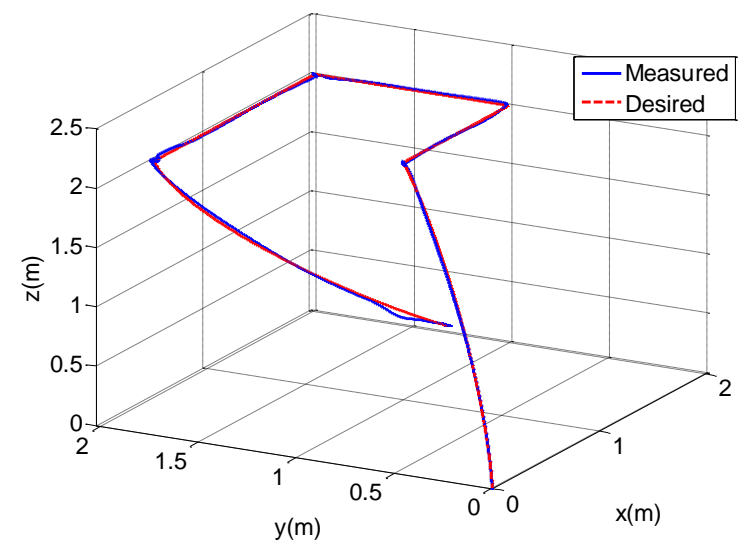

Figure 8. Absolute position of the octorotor with disturbances and parametric variations (case b)
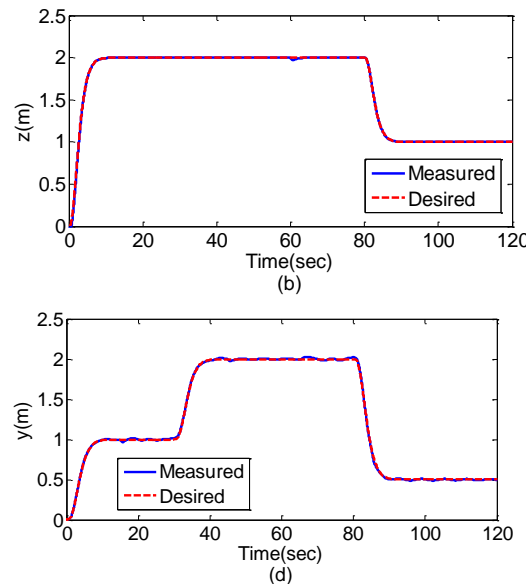

Figure 9. Trajectory of the output variables
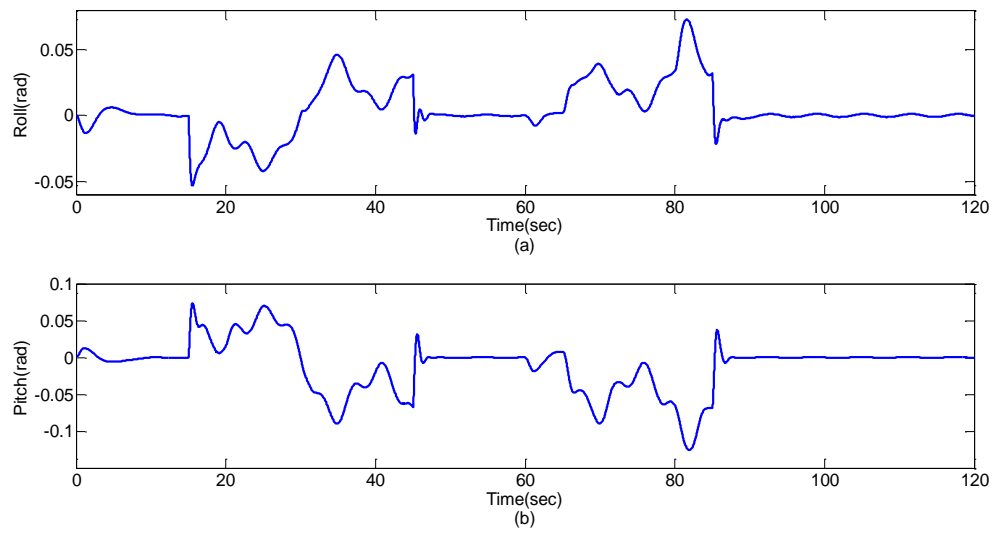

Figure 10. Trajectory of roll and pitch 


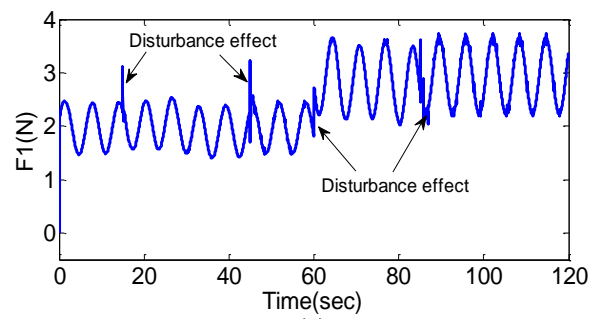

(a)

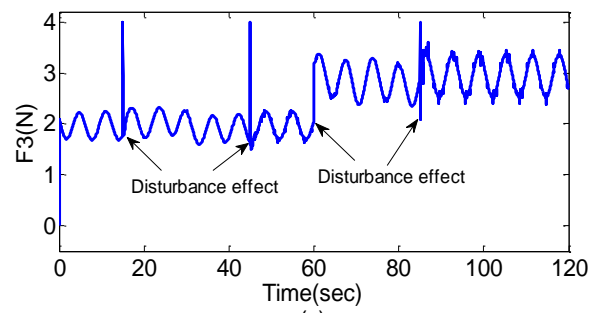

(c)
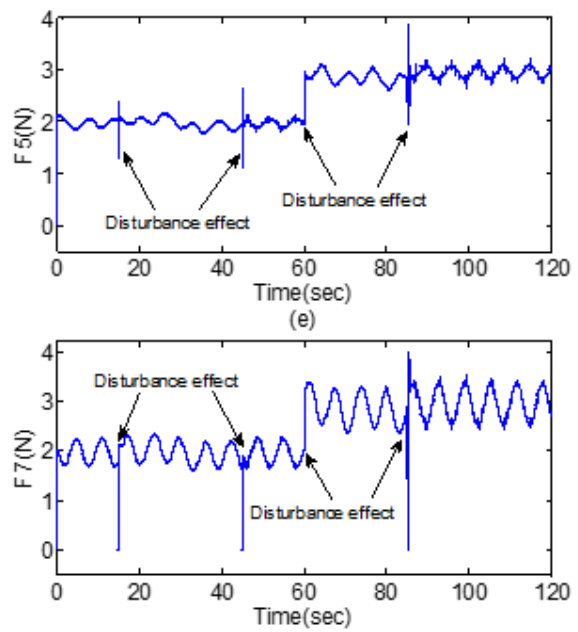

(g)

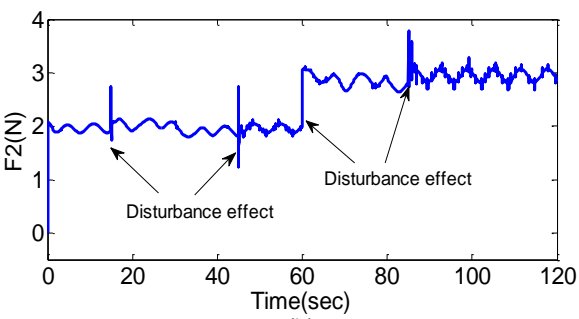

(b)

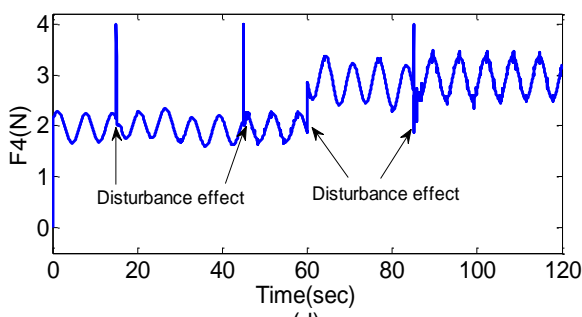

(d)
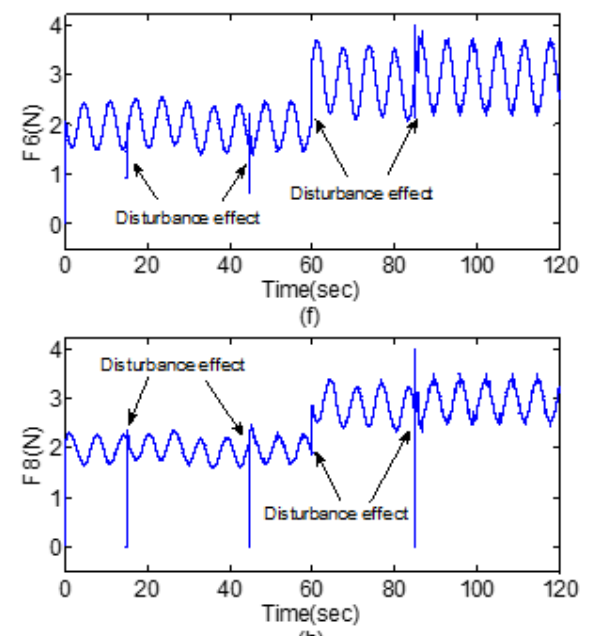

(h)

Figure 11. Control inputs

\section{CONCLUSIONS}

In this paper, the robust adaptive controller that is based on type-1 and type- 2 fuzzy systems has been investigated. In this controller, the adaptive fuzzy control law ensures the convergence of tracking errors and all signal plants in presence the external disturbances and parametric variations. This law incorporates adaptive parameters to compensate the external disturbances effect and the reconstruction errors. The application of the developed method is carried out for a coaxial octorotor. The obtained simulation results show that this robust adaptive control type- 1 and type- 2 fuzzy law maintains the tracking errors in an acceptable interval in the presence of extreme or significant parameter variations or external disturbances. Because of the simple nature of the proposed approach, it is easy to implement in real-time control systems. In addition the comparative study performed with other works developed in the literature, has shown the effectiveness of the proposed control approach. In the future work the experimental implementation of the proposed control scheme will be addressed. The future works should synthesize non linear observer to deal with unmeasured states. It should consider actuator faults and developing a fault tolerant controller based on control design proposed in this paper. As will as should envisage the experimental implementation of the proposed control scheme.

\section{REFERENCES}

[1] Salih AL, Moghavvemi M, Mohamed AF. (2010). Flight PID controller design for a UAV quadrotor. Scientific Research and Essays 23(5): 3660-3667.

[2] Utkin VI. (2008). Sliding modes in control and optimization. Berlin, Germany: Springer-Verlag https://doi.org/10.1007/978-3-642-84379-2 6

[3] Besnard L, Shtessel YB, Landrum B. (2011). Quadrotor vehicle control via sliding mode controller driven by sliding mode disturbance observer. Journal of the Franklin Institute $349(2)$ : $1-27$. https://doi.org/10.1016/j.jfranklin.2011.06.031

[4] Das A, Lewis F, Subbaro K. (2009). Backstepping approach for controlling a quadrotor using lagrange form dynamics. J. Intell. Robot. Syst. 56(1): 127-151. https://doi.org/10.1007/s10846-009-9331-0

[5] Zemalache K, Maaref H. (2009). Controlling a drone: Comparison between a based model method and a fuzzy inference system. Appl. Soft Comput. 9(2): 553-562. https://doi.org/10.1016/j.asoc.2008.08.007

[6] Nicol C, Macnab CJB, Ramirez-Serrano A. (2008). Robust neural network control of a quadrotor helicopter. In: Proceeding of the IEEE international conference Electrical and Computer Engineering. Niagara Falls, 
[7] Peng C, Bai Y, Gong X, Gao QJ, Zhao CJ, Tian YT. (2015). Modeling and robust backstepping sliding mode control with adaptive RBFNN for a novel coaxial eightrotor UAV. IEEE/CAA Journal of Automatica Sinica 2: 56-64. https://doi.org/10.1109/JAS.2015.7032906

[8] Wai RJ, (2007). Fuzzy sliding mode control using adaptive tuning technique, IEEE Trans. Indus. Elect 54: 586-594. https://doi.org/10.1109/tie.2006.888807

[9] Karnik NN, Mendel JM, Liang Q. (1999). Type-2 fuzzy logic systems. IEEE Transactions on Fuzzy Systems 7(6): 643-658. https://doi.org/10.1109/91.811231

[10] Singh A, Jha M, Qureshi MF. (2014). Design of genetically tuned interval type-2 fuzzy PID controller for load frequency control (LFC) in the un-regulated power system. AMSE Journals -2014-Series: Advances C 69(1): 85-104.

[11] Wu D, Tan W. (2006). A simplified type-2 fuzzy logic controller for real-time control. ISA Transactions 45(4):
503-510. https://doi.org/10.1016/S0019-0578(07)602286

[12] Castillo O, Marroquín M, Melin P, Valdez F, Soria J. (2012). Comparative study of bio-inspired algorithms applied to the optimization of type-1 and type-2 fuzzy controllers for an autonomous mobile robot. Information Sciences 192:

19-38. https://doi.org/10.1016/j.ins.2010.02.022

[13] Alwi H, Edwards C. (2013). Fault tolerant control of an octorotor using LPV based sliding mode control allocation. In: Proceedings of the American Control Conference. Washington, 6505-6510. https://doi.org/10.1109/ACC.2013.6580859

[14] Majd S, Benjamin L, Isabelle F, Clovis F, Hassan S, Guillaume S. (2015). Fault diagnosis and fault-tolerant control strategy for rotor failure in an octorotor. In: Proceedings of the IEEE International Conference on Robotics and Automation. Seattle 5266-5271. https://doi.org/10.1109/ICRA.2015.7139933 